\title{
Time-varying Inflation Dynamics and Monetary Policy Credibility: A Bivariate Approach with Stochastic Uncertainty
}

\author{
Ning Zeng \\ School of Business, Macau University of Science and Technology, Macau, China \\ nzeng@must.edu.mo
}

Keywords: Inflation, Credibility, Stochastic Uncertainty.

\begin{abstract}
This paper addresses the properties of inflation dynamics and proposes a univariate ARFIMA-EGARCH-in-mean model to accommodate inflation rates, expectations, volatilities and persistence. As the changes within inflation dynamics may be caused by the lack of monetary policy credibility, a generalized approach is developed to be concerned with the measurement of credibility. Through a bivariate model, all possibilities for relevant variables and effects can be capsuled in one framework simultaneously. And thus empirical results should deliver useful implications should for the conduct of central banks' monetary policy.
\end{abstract}

\section{Introduction}

The macroeconomic variable of inflation - a sustained rise in the general level of prices - describes the purchasing power of money and the real value of the money. With a stable price level, it is conducive to reducing businesses' and households' fear of declining future purchasing power and lowering both nominal and real interest rates. And thus it encourages investment, improves productivity and leads ultimately to sustainably high levels of economic growth and employment. Therefore understanding the property of inflation process would be a reliable method to reflect economic efficiency.

Since the Second World War, many monetary authorities have had set price stability gradually as the primary goal $[1,2]$, although a downward trend did not gain real momentum until the 1980s. Exogenous events may have caused this downward trend, such as the Vietnam War (1966-1974), the collapse of the Bretton Woods Agreement on fixed exchange rates (1971), the oil price shocks (1973-1974, 1979) and financial crisis (2008).

Notably, the US-the biggest single economy of the world-has not been experiencing price stability always. From the end of 1940s to the middle of 1960s, the annual US inflation averaged 1.6\%. This period is described as postwar prosperity. In the following period until the early 1980s, the average annual inflation rate has increased to a peak of $7 \%$ or so. What has become known as the Great Inflation had several causes, including the successive oil price shocks of 1973-1974 and 1979. Then the US inflation rates experienced a dramatic drop to around $2 \%$ in 1986, and the average annual inflation rate has since remained at 3\%. However, inflation has been down at $1.7 \%$ since the financial crisis in 2008.

In 1977, Friedman explained high inflation may result in irregular policy responses to curb it, and consequently increase uncertainty. Cukierman and Meltzer also demonstrated that higher inflation uncertainty could raise the level of inflation rate in their work (1986). Whereas Holland argued that there is an adverse relationship between inflation uncertainty and inflation in 1995, since policymakers consider an increase in inflation as a cost, and hence will take action to reduce inflation in the future.

Moreover, studies have drawn intensive interest of inflation persistence-the speed of the responses of inflation to shock, which is one of the most controversial topic on monetary policy. For example, it is widely agreed that inflation persistence was very high from 1965 to the early 1980s. Then low inflation persistence occurred in the 1947-1959 period and the 1960s and followed by the 
Volcker-Greenspan era as well, which favours the view that inflation tends to return to its mean after a quick adjusting shift following a shock. Other empirical suggested that the US inflation has been highly persistent during the postwar period [3], approaching that of a random walk process. Also in their work of 2007, Pivetta and Reis found that US inflation is best described as high and time invariant since 1965 .

One reason to explain why inflation process is persistent rather than occasional and temporary, is the lack of monetary policy credibility [4]. More importantly, the degree of inflation persistence are time varying. This paper captures the properties of inflation dynamics and proposes a new measurement of credibility, which may deliver significant implications for the conduct of monetary policy.

\section{Inflation Volatility, Expectations and Persistence}

Since the complex interactions among inflation, volatility and persistence has not been extensively discussed in the literature, this paper proposes a framework to accommodate inflation rate, volatility, expectations and persistence simultaneously rather than individually.

One solution to this issue is employing a unilabiate exponential GARCH (EGARCH) model of Nelson of 1991, which does not impose parameter restrictions to ensure positivity of the conditional variances and allows an asymmetric response to shocks. Meanwhile, in the mean equation, Baillie et al. introduced a general property of an ARFIMA process in 1996, depicting inflation expectations (the autoregressive terms) and persistence (the parameter of $d$ ). The ARFIMA (n, $d$, and m)-EGARCH (p, q) is written as:

$$
\begin{gathered}
\phi(L)(1-L)^{d}\left(\pi_{t}-\mu\right)=\delta(L) h_{t}^{1 / 2}+\varepsilon_{t} \\
\ln h_{t}=\omega_{0}+\alpha(L) g\left(z_{t}\right)+\beta(L) \ln h_{t}+\gamma(L) \pi_{t-1} \\
g\left(z_{t}\right)=\theta_{1} z_{t}+\theta_{2}\left[\left|z_{t}\right|-E\left|z_{t}\right|\right]
\end{gathered}
$$

where $\pi_{t}$ is inflation, $h_{t}$ is the variances, $\mu$ is the intercept, $z_{t} \sim$ iid $(0,1)$; $L$ is the lag operator, $d$ is the inflation persistence driving factor, which is between zero and unity; $\phi(L)=1-\sum_{i=1}^{n} \phi_{i} L^{i}$, $\alpha(L)=\sum_{i=1}^{q} \alpha_{i} L^{i}, \beta(L)=\sum_{i=1}^{p} \beta_{i} L^{i}$, and all the roots of $\phi(L), \beta(L)$ and $\alpha(L)$ lie outside the unit circle; $\delta$ shows how the level of the inflation rate is affected by its volatilities, and $\gamma$ expresses the impacts of inflation on its volatility. The innovations $\varepsilon_{t}$ are assumed $h_{t}^{1 / 2}$ with appropriate distributions (Gaussian, Student's t, and some others) conditional on information set up to time $t$ - 1 , following an EGARCH process.

In addtion, when $d=0$, the series follows an $\mathrm{I}(0)$ process with short memory, implying a quick regain of its equilibrium after a shock; when $d=1$, the series does not revert to its mean and the persistence of shocks is infinite; when $0<d<1$, and $\mathrm{I}(d)$ process with long-run dependence, in which persistence dies out hyperbolically, that is, the series takes a considerable time to reach mean reversion aftershocks.

Equation (1)-(3) is denoted as an ARFIMA-EGARCH-in-mean model, which is able to capture the inflation dynamics, expectations, volatility, the degree of persistence, and meanwhile allow inflation and its volatility to affect each other. Hence it is adequate to examine interactions among inflation, expectations, volatility and persistence.

\section{Then Measurement of Credibility}

If monetary authorities have single goal of monetary policy-inflation target, the measurement of the credibility is involved with inflation only. In 1989, Hardouvelis and Barnhart modelled the response of commodity price-underlying inflation to weekly M1 data releases, applying a random 
coefficients Kalman filter to examine the evolution of the Federal Reserve (the Fed) credibility. They suggested that the announcement of targeting M1 to reduce inflation in November 1979 did not help the Fed with restoring credibility immediately, and that credibility was established slowly as inflation fell to low levels by the mid-1980s. Another findings of their study is that policy results, instead of policy announcements, were the real focus of economic agents. Thus credibility may vary with the underlying inflation.

Another measures of credibility is to create credibility variables. Under the assumption of a credible policy, the public's inflation expectations tend towards an inflation target with little deviation. If there is an explicitly numeric inflation target, the gap between the target and public expectations could be acted as a measure of the credibility [5]. Without explicit inflation targets, see, $2 \%$, this measure could be more complicated and yet unconvincing. In 1994, Croushore and Koot created two variables of the Fed's credibility by taking the difference between the Fed's forecast and public expectations, and the Fed's forecast and the actual inflation. They found that the Fed credibility was "fairly high in the late 1970s and early 1980s" (p. 229).

As for the Fed with a dual mandate-maintaining price stability and maximising employment, existing approaches would not be adequate. It is wise to consider that not only inflation but also unemployment rate should be included in one model. Apart from the disputable Phillips curve-the inverse relation between inflation and unemployment, a bivariate model, capsuling inflation and unemployment, may be more suitable for to assess the Fed's monetary policy credibility.

Let $\pi_{t}$ and $U_{t}$ denote the dynamics of inflation and unemployment respectively, a bivariate (B)$\operatorname{ARFIMA}(\mathrm{n}, d, \mathrm{~m})-\operatorname{EGARCH}(\mathrm{p}, \mathrm{q})$-in-mean model is written as:

$$
\begin{aligned}
& \Phi(L) P^{d}\left(x_{t}-\mu_{0}\right)=\Delta(L) h_{t}^{1 / 2}+\varepsilon_{t} \\
& \ln h_{t}=\omega_{0}+\alpha(L) g\left(z_{t}\right)+\beta(L) \ln h_{t}+\Gamma(L) x_{t-1} \\
& g\left(z_{t}\right)=\theta_{1} z_{t}+\theta_{2}\left[\left|z_{t}\right|-E\left|z_{t}\right|\right] \\
& h_{\pi s, t}= \rho \sqrt{h_{\pi t}} \sqrt{h_{s t}}, \quad-1 \leq \rho \leq 1
\end{aligned}
$$

Where $\varepsilon_{t}=\hat{H}_{t}^{1 / 2} z_{t}, \hat{H}_{t}=\operatorname{diag}\left(h_{t}\right), z_{t} \sim \operatorname{iid}(0, \mathrm{C})$, and $C$ is a $2 \times 2$ fixed correlation matrix with units on the diagonal. $x_{t}=\left[\begin{array}{l}\pi_{t} \\ u_{t}\end{array}\right], \mu_{0}=\left[\begin{array}{l}\mu_{\pi} \\ \mu_{u}\end{array}\right], h_{t}=\left[\begin{array}{l}h_{\pi t} \\ h_{u t}\end{array}\right], \varepsilon_{t}=\left[\begin{array}{l}\varepsilon_{\pi t} \\ \varepsilon_{u t}\end{array}\right], z_{t}=\left[\begin{array}{l}z_{\pi t} \\ z_{u t}\end{array}\right], \Phi=\left[\begin{array}{ll}\phi_{\pi \pi} & \phi_{\pi u} \\ \phi_{u \pi} & \phi_{u u}\end{array}\right]$, $\Delta=\left[\begin{array}{ll}\delta_{\pi \pi} & \delta_{\pi u} \\ \delta_{u \pi} & \delta_{u u}\end{array}\right], \Phi(L)=I-\sum_{i=1}^{n} \Phi_{i} L^{i}, I$ is a $2 \times 2$ identity matrix. $P^{d}$ Is a $2 \times 2$ diagonal matrix with elements $(1-L)^{d_{\pi}}$ and $(1-L)^{d_{u}}$, with $d_{\pi}$ and $d_{u}$ measuring the long memory of inflation and unemployment respectively. $x_{t}$ Is the column vector of inflation and unemployment, $\mu_{0}$ denotes the intercepts and $h_{t}$ is the variances. $\Phi$ Is the coefficient matrix of the vector autoregressive component capturing the mutual effects between inflation and unemployment? $\Delta$ Captures the in-mean effects implying how the level rates of the two variables are affected by their volatilities. $\varepsilon_{t}$ is the innovations assumed to be serially uncorrelated, normally/student's t/others distributed with mean vector 0 , variance $h_{t}$ and covariance $h_{\pi u, t}$ conditional on information set up to time $t$ - 1 , while $h_{t}$ is formed following the ccc EGARCH process.

$$
\omega_{0}=\left[\begin{array}{l}
\omega_{\pi} \\
\omega_{u}
\end{array}\right], A=\left[\begin{array}{ll}
\alpha_{\pi \pi} & \alpha_{\pi u} \\
\alpha_{u \pi} & \alpha_{u u}
\end{array}\right], B=\left[\begin{array}{ll}
\beta_{\pi \pi} & \beta_{\pi u} \\
\beta_{u \pi} & \beta_{u u}
\end{array}\right], \Gamma=\left[\begin{array}{ll}
\gamma_{\pi \pi} & \gamma_{\pi u} \\
\gamma_{u \pi} & \gamma_{u u}
\end{array}\right] . \quad \Gamma \text { Captures the impacts of the }
$$

level rates of both variables on their volatilities. $g=\left[\begin{array}{c}g_{\pi} \\ g_{u}\end{array}\right], \theta_{1}=\left[\begin{array}{c}\theta_{1 \pi} \\ \theta_{1 u}\end{array}\right]$ and $\theta_{2}=\left[\begin{array}{c}\theta_{2 \pi} \\ \theta_{2 u}\end{array}\right]$. $h_{t}$ In equation

(5) will be almost surely covariance stationary if $\theta_{1 \pi} / \theta_{1 u}$ and $\theta_{2 \pi} / \theta_{2 u}$ do not both equal zero, and positive definite for all $t$ allowing $\Gamma$ to reflect positive/negative influences of inflation and 
unemployment on their variances. $\theta_{1}$ Captures the leverage effects when $\theta_{1}<0$ and $\ln h_{t}$ responds symmetrically to $z_{t}$ when $\theta_{1}=0$.

Equation (4)-(7) is a bivariate version of Equation (1)-(3), providing a flexible solution to accommodating the Fed's dual-goal, while allowing all the possible interactions among the two variables as well as their volatilities. This approach also depicts the properties of persistence of both variables, which has been neglected in the literature, so that it is a reliable to be employed for an assessment of the Fed's monetary policy credibility.

\section{Conclusion}

This paper has been concerned with the properties of inflation dynamics, including expectations, volatilities and persistence, together with the measurement of the Fed's monetary policy credibility. Since inflation target may not be the only goal for some monetary authorities such as the Fed, a generalised model is developed to accommodate all possibilities for relevant variables and effects. Through this approach, monetary policy credibility could be assessed while capturing characters of dynamics, and empirical results should deliver useful implications for the conduct of central banks' monetary policy.

\section{References}

[1]. Samuelson, P. A., Nordhaus, W. D., Economics (18th Edition). McGraw-Hill, 2004.

[2]. Mankiw, N. G., Macroeconomics (6th Edition), Worth Publishers, 2007.

[3]. Fuhrer, J., Moore, G., Inflation Persistence, The Quarterly Journal of Economics, 1995, p. 127-159.

[4]. Fellner,W., Towards a Reconstruction of Macroeconomics: Problems of Theory and Policy, American Enterprise Institute for Public Policy Research, Washington, D.C., 1976.

[5]. Tanuwidjaja, E., Choy, K. M., Central bank credibility and monetary policy in Indonesia, Journal of Policy Modeling. Vol. 28 (2006) No.9, p. 1011-1022. 